\title{
'We like it wet': A comparison between dissection techniques for the assessment of parity in Anopheles arabiensis and determination of sac stage in mosquitoes alive or dead on collection
}

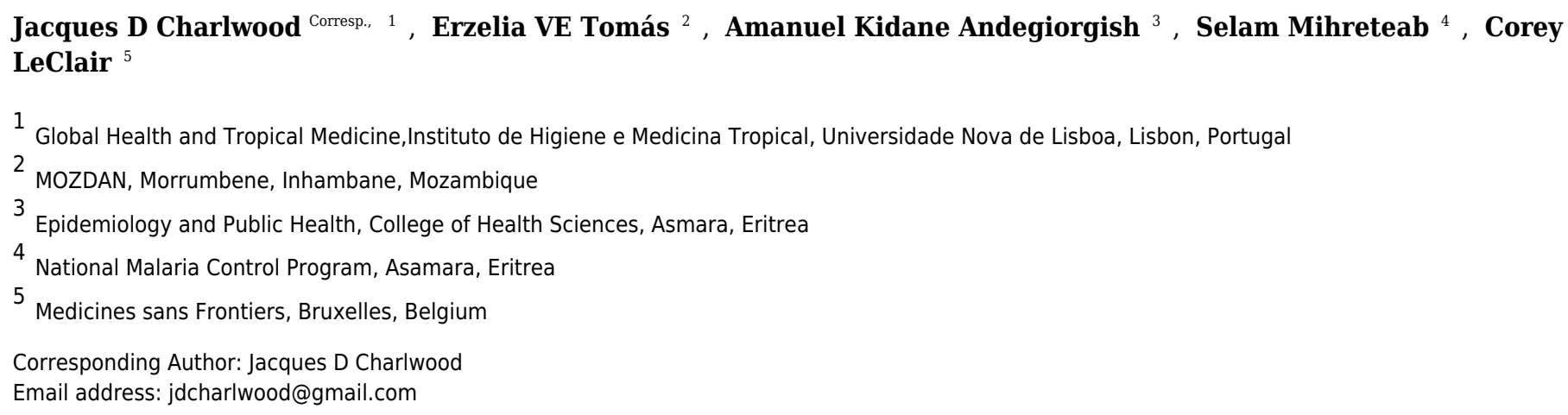

Background. The determination of parous rates in mosquitoes, despite numerous shortcomings, remains a tool to evaluate the effectiveness of control programs and to determine vectorial capacity in malaria vectors. Two dissection techniques are used for this. For one, the tracheoles of dried ovaries are examined with a compound microscope and in the other the follicular stalk of ovaries is examined, wet, with a stereomicroscope. The second method also enables the sac stage of parous insects (which provides information on the duration of the oviposition cycle) and mated status of insects to be determined. Despite widespread use the two techniques have not previously been compared. Methods We compared the two dissection techniques using Anopheles arabiensis, collected with a tent-trap in Eritrea. The paired ovaries were removed in water and one was examined by each method. From a separate set of dissections from Tanzania, we also determined if the sac stages of $A$. gambiae s.l. (83\% of 183 identified by PCR being Anopheles arabiensis the remainder being $A$. gambiae) that were alive on collection were different to those that died on collection and what the implications for vectorial capacity estimation might be. Results Seven per cent of the dry ovaries could not be classified due to granulation (yolk) in the ovariole that obscured the tracheoles.. The sensitivity of the dry dissection was $88.51 \%$ (C.I. 79.88-94.35\%) and the specificity was $93.55 \%$ (C.I. 87.68-97.17\%) among the 211 ovaries that could be classified by the dry technique and compared to the ovaries dissected wet. 1823 live and 1416 dead from Furvela tent-traps, CDC light-trap and window-trap collections were dissected 'wet' from Tanzania. In these collections parous insects were more likely to die compared to nulliparous ones. The 
proportion of parous mosquitoes with 'a' sacs (indicative of recent oviposition) was significantly greater in insects that were dead (0.36) on collection in the morning compared to those that were alive $(0.12)$ (Chi square $138.93, p<0.001$ ). There was a preponderance of newly emerged virgin insects in the outdoor collection (Chi sq $=8.84, p=$ 0.003). Conclusions In anophelines the examination of mosquito ovaries using transmitted light in a 'wet' dissection is a more useful and informative technique than examination of dry ovaries. In order to correctly estimate the duration of the oviposition cycle mosquitoes should be dissected as soon as possible after collection. Younger insects were more likely to attempt to feed outdoors rather than indoors. 
1 A comparison between 'wet' and 'dry' dissections for the assessment of parity in

2 Anopheles arabiensis and determination of sac stage in mosquitoes alive or dead on

3 collection.

4

5 JD Charlwood ${ }^{1,2}$, EVE Tomás ${ }^{3}$, AK Andegiorgish ${ }^{1}, \mathrm{~S} \mathrm{Mihreteab}^{4}$ and C LeClair ${ }^{5}$.

6

7 1-College of Health Sciences, Asmara, Eritrea

8 2- Global Health and Tropical Medicine, Instituto de Higiene e Medicina Tropical Universidade

9 NOVA de Lisboa Rua da Junqueira n. ${ }^{\circ} 100$ | 1349-008 Lisboa

10

11

12

13

16

17

18

22

23

3-MOZDAN, PO Box 8, Morrumbene, Mozambique

4- Malaria Control Program, Asmara, Eritrea.

5-Medicens sans Frontiers, Bruxelles, Belgium.

Email:

jdcharlwood@gmail.com

elsatomas007@gmail.com

akidane2016@gmail.com

selamino2001@yahoo.com

corey_leclair@hotmail.com

correspondence: jdcharlwood@gmail.com 
24

25

26

27

28

29

30

31

32

33

34

35

36

37

38

39

40

41

42

43

44

45

46

47

48

49

50

51

52

53

54

55

56

57

58

59

60

61

62

63

64

65

\section{Abstract}

Background. The determination of parous rates in mosquitoes, despite numerous shortcomings, remains a tool to evaluate the effectiveness of control programs and to determine vectorial capacity in malaria vectors. Two dissection techniques are used for this. For one, the tracheoles of dried ovaries are examined with a compound microscope and in the other the follicular stalk of ovaries is examined, wet, with a stereomicroscope. The second method also enables the sac stage of parous insects (which provides information on the duration of the oviposition cycle) and mated status of insects to be determined. Despite widespread use the two techniques have not previously been compared.

Methods We compared the two dissection techniques using Anopheles arabiensis, collected with a tent-trap in Eritrea. The paired ovaries were removed in water and one was examined by each method. From a separate set of dissections from Tanzania, we also determined if the sac stages of A. gambiae s.1. (83\% of 183 identified by PCR being Anopheles arabiensis) that were alive on collection were different to those that died on collection and what the implications for vectorial capacity might be.

Results 389 host-seeking, mosquitoes, from Furvela tent-traps in Eritrea and 1823 live and 1416 dead from Furvela tent-traps, CDC light-trap and window-trap collections were dissected from Tanzania. Seven per cent of the dry ovaries could not be classified due to granulation (yolk) in the ovariole that obscured the tracheoles. The sensitivity of the dry dissection was $92.74 \%$ (C.I. $86.67-96.63 \%$ ) and the specificity was $88.51 \%$ (C.I. 79.88-94.35\%) among the 211 ovaries that could be classified by the dry technique and compared to the ovaries dissected wet. In collections from Tanzania parous insects were more likely to die compared to nulliparous ones. The proportion of parous mosquitoes with 'a' sacs (indicative of recent oviposition) was significantly greater in insects that were dead $(0.36)$ on collection in the morning compared to those that were alive $(0.12)$ (Chi square $138.9259, \mathrm{p}<0.001)$. There was a preponderance of newly emerged virgin insects in the outdoor collection $($ Chi $\mathrm{sq}=8.8413, \mathrm{p}=0.003)$.

Conclusions The examination of mosquito ovaries using transmitted light in a 'wet' dissection is a more useful and informative technique than examination of dry ovaries. In order to correctly estimate the duration of the oviposition cycle mosquitoes should be dissected as soon as possible after collection. Younger insects were more likely to attempt to feed outdoors rather than indoors.

\section{Introduction}

Despite its many shortcomings the measurement of parous and nulliparous rates (i.e. the proportion of insects in a population that have, or have not, laid eggs) in mosquito vectors is commonly evaluated as part of malaria control programs. The shortcomings include the requirement that measurements are made over a complete population cycle, that nulliparous and parous insects are sampled without bias and (for survival rate estimation) that survival is 
66 independent of age (Clements \& Paterson, 1981). Although they may not provide much more

67 than an approximation of survival (Gillies, 1989), an estimation of parous rates is useful in

68 control trials for comparisons between intervention and control areas (where they are expected to

69 be lower in interventions that target adult mosquitoes but higher in those that target larvae) and,

70 independent of survival estimation, they also provide information on the behaviour of young

71 insects that may themselves become a target for specific interventions.

72

73 Parity is determined by dissection. Following the maturation of the first batch of eggs

74 irreversible changes occur in the ovaries of female mosquitoes. The tightly packed and coiled

75 tracheolar system characteristic of nulliparous insects, and illustrated in publications of the WHO

76 (WHO,2013), becomes stretched and uncoiled as the eggs develop and never return to their

77 previous state (Detinova, 1962). In newly emerged teneral mosquitoes' meconium, the remains

78 of larval midgut epithelium can be seen as a green an opaque mass inside the midgut

79 (Rosay,1961 Romoser et al. 2000). This is excreted either following an initial blood meal or

80 within $48 \mathrm{hrs}$ of emergence. The tracheolar system can be seen in ovaries that are dissected in

81 distilled water and allowed to dry. Once dry the ovary can be examined under a compound

82 microscope. The 'dry' technique is simple and has been widely used.

83

84 An alternative technique is to examine the follicular stalk at the time of dissection with

85 transmitted light using a stereomicroscope. The dissection is performed in isotonic saline (to 86 avoid swelling of tissues) but can also be performed with water. This dissection has the 87 advantage that it can provide information on both the mated status and the duration of the 88 oviposition cycle in parous insects 
89

90 Irreversible changes also occur following oviposition in the pedicel that connects the ovarioles to

91 the lateral oviduct (Hoq \& Wilkes, 1995). In this case granulation occurs in the basal body,

92 small areas in the calyx wall enclosed by the ovariolar sheaths consisting of six to nine 93 specialized epithelial cells, making them visible when examined with light coming through the

94 preparation. A large egg sac remains in the ovarioles immediately after ovulation. The sac 95 gradually contracts and, 12-24 h after ovulation, consists of heavily folded tunica above the

calyx. In nulliparous females, there is no coloration of the pedicel. The tracheolar system is visible in this dissection so that its appearance can also assist in interpretation of the preparation. Examination of the pedicel in parous females also enables the duration of the oviposition cycle to be estimated since an insect with a large sac would have been caught shortly after oviposition whereas one with just a basal body would have oviposited approximately a day earlier. The duration of the oviposition cycle has a major impact on the proportion of mosquitoes that might be vectors. For example, a change from a two-day cycle to a three-day one produces a four-fold increase in the potential numbers of vectors.

The dissection also enables the mated status of newly emerged nulliparous insects to be determined. In particular, examination of the spermatheca and oviducts is possible. Virgin insects do not have sperm in the spermatheca and, in recently mated anophelines, there is a male donated mating plug (Gillies 1956, Baldini et al., 2013) visible in the common oviduct. This is absorbed over the following 12-24 hours. Thus, with this dissection it is possible to separate nulliparous insects into three categories: virgins, recently mated insects and those that have mated 24 hours, or more, earlier (Charlwood et al., 1985, 1986a,b,c, 2003, 2011, Charlwood \& Tomás, 2011) . In practise, the overall appearance of the ovary is used to assess parous status: 
113 transparent and small ovaries with coiled tracheoles are indicative of nulliparity, whilst larger,

114 darker, ovaries with enlarged ampullae (Gillies, 1956) and an uncoiled tracheolar system indicate

115 that the mosquito is parous.

116

117 Anopheleines and Aedines differ in that in the former oogenesis is an 'all or nothing'

118 phenomenon that requires a complete blood meal to proceed whilst in the latter, individual

119 follicles may develop following partial blood meals. This makes estimates of age more difficult

120 in the latter group.

121 Hugo et al (2008) compared these and more sophisticated techniques using laboratory reared

122 Aedes vigilax and Culex annulirostris. They considered that the dry technique (when allied to

123 the observation of the presence of meconium in the stomach of the mosquito) was the most

124 suitable for parity determination.

125 The two methods of dissection have not previously been compared in anophelines, nor have they 126 been compared using wild insects whose life conditions differ from insects reared and 127 maintained in the laboratory. Here, therefore, we compare these methods with wild caught 128 Anopheles arabiensis. We also compare estimates of the duration of the oviposition cycle from 129 insects that died shortly after collection with those that remained alive up to the time of 130 dissection.

131 Methods

132 Description of study sites

133

134 Anopheles arabiensis, collected on 17 nights between the $7^{\text {th }}$ and $23^{\text {rd }}$ of October 2017 with a 135 Furvela tent-trap (Charlwood et al., 2017) below the village of Adi Bosco (15 41' 41.67" N 38 136 38' 54.59' E at an altitude of 1536m above sea level) in Anseba province, Eritrea, were dissected 137 in bottled drinking water. Mosquitoes, that were alive upon collection, were killed in a freezer 
138 and were then used for these dissections. One ovary was placed on a slide to dry for subsequent

139 examination and the other was assessed directly for parity and sac stage. Insects from the latter

140 dissection were classified according to the scheme outlined by Charlwood et al., (2003). The sac

141 stage in parous insects was determined according to the scheme outlined in Wilkes \& Charlwood 142 (1975) and illustrated in Figure 1.

143 Each mosquito was given a unique identifying number and subsequently comparisons between 144 assessments of parity were determined. A number of Culex quinquefascaitus collected with a 145 CDC light-trap from a bedroom in Asmara, Eritrea, where potential hosts slept under mosquito 146 nets were also dissected for a comparison of the appearance of the ovaries of the two species.

148 The sac stages of mosquitoes according to whether they were alive or dead upon collection were 149 determined from 42 nights of collection undertaken in the village of Kyamyorwa in Muleba 150 district, Kagera Province, Tanzania, from December 12015 to January 17 2016. Mosquitoes 151 were collected in a CDC light-trap, run inside a bedroom with two human and one canine host; a 152 window-trap from the same room and a Furvela tent-trap outdoors with a single sleeper (Le Clair 153 et al., 2017). Live mosquitoes were removed from the collection bags with an aspirator prior to 154 being killed and both recently killed and those dead on collection were identified to species or 155 species group using the keys of Gillies \& De Meillon (1968) and Gillies \& Coetzee (1975). 156 Mosquitoes in Tanzania were dissected in saline eye drops (Charlwood et al., 2016).

157 Anopheles arabiensis is the only member of the A. gambiae complex that has been identified 158 from previous collections in Eritrea (Shilulu et al., 2003) and so it is assumed that this was the 159 member of the complex that was collected. A sub-sample of the A. gambiae s.l. from 
160 Kyamyorwa were identified to species by multiplex real-time PCR Taq Man assay (Bass et al., 161 2008).

162 In order to determine if the different age groups were caught in similar proportions indoors (in

163 light-trap and window-trap combined) and outdoors (in the tent-trap) the number of the different 164 ages collected live and dead were estimated by multiplying the total by the proportion in each 165 category and then summing the estimated totals. The overall proportion of each age group 166 (indoors and outdoors) was then estimated and indoor and outdoor collections compared by Chi167 Square test (at a significance level of 0.05).

168 We also assume that gonotrophic development (from blood feeding to becoming gravid) takes 2 169 days in Kyamyorwa; hence mosquitoes with 'a' or 'b' sacs (Sac) were considered to have a 2170 day feeding cycle and those with 'c' or 'd' (No-sac) to have added an extra day (i.e. to have a 3171 day cycle). Estimates of the population mean duration of the feeding cycle $(\mu)$ in live and dead 172 parous insects were therefore determined according to the proportions of Sac and No-sac 173 mosquitoes in the collection where $\mu$ is the mean feeding frequency of parous insects in days:

174

175

176

177 178 179 180

181

182

183 The collections conducted in Tanzania were done as a component of the Pan African Malaria 184 Vector Research Consortium project 'Evaluation of a novel long lasting insecticidal net and 185 indoor residual spray product, separately and together, against malaria transmitted by pyrethroid 
186 resistant mosquitoes' which received ethical clearance from the ethics review committees of the

187 Kilimanjaro Christian Medical College (certificate number 781 on the 16 September 2014), the

188 Tanzanian National Institute for Medical Research (20 August 2014), and the London School of

189 Hygiene and Tropical Medicine (reference 6551 on 24 July 2014). The trial was registered with

190 ClinicalTrials.gov (registration number NCT02288637) on 11 July 2014.

191 Collections in Eritrea were undertaken by the first author in his tent during supervision of 192 students from the College of Health Sciences, Asmara, undertaking their fieldwork as part of a 193 course entitled 'The ecology of malaria vectors'.

194

195

196

197

198

199

200

201

202

203

204

205

206

207 208

209 210

\section{Results}

Ovaries of $C x$. quinquefasciatus were clearer and the tracheoles easier to see than was the case with the $A$. arabiensis (compare Figures 2A, B with Figures 3A, B).

In almost $10 \%$ (23 of 238 ) of the A. arabiensis dissected dry the deposition of yolk in the follicles made assessment of the age difficult or impossible from the dry dissections. In some cases, wetting the preparation again temporarily enabled the tracheoles to become visible for assessment (Fig 4A and B).

\section{Comparison between methods}

There were 211 ovaries that could be classified by the dry technique and compared to the ovaries dissected wet (Table 1). There was a 91.5\% (C.I. 86.30-94.49\%) concordance between the methods. Nevertheless, 18 of 211 (10 parous and 8 nulliparous) were given different classifications by the two methods. Thus, assuming that the wet dissection was the 'gold standard', the sensitivity of the dry dissection was $88.51 \%$ (C.I. 79.88-94.35\%) and the specificity was $93.55 \%$ (C.I. 87.68-97.17\%). 
211 During the experiment, the number of A. arabiensis collected decreased from a mean of 126 per

212 tent per night to 34 per night whilst the parous rate increased from 0.28 to 0.56 (correlation

213 between the number collected and parous rate $=-0.71$ ). Since the population was changing and

214 collections did not cover the complete population cycle any estimates of survival from the

215 present data would be imprecise and possibly incorrect.

216 Sac stages among live or dead mosquitoes

217

218 Among 183 A. gambiae s.l. from Kyamyorwa identified to species by PCR 152 (83.1\%) were $A$.

219 arabiensis and the remainder were $A$. gambiae (LeClair et al., 2017). Thus, the great majority of

220 insects from Kyamyorwa were also A. arabiensis.

221 Between November 302015 and January 17 2016, 1823 live (273 from the light- trap, 341 from

222 the window trap and 1209 from the tent-trap) and 1416 dead (711 from the light-trap and 705

223 from the tent-trap) A. gambiae s.l. were dissected (Table 2). The smaller numbers of live insects

224 dissected from the light-trap was due to the low survival of the mosquitoes in the trap (LeClair et 225 al, 2017). All insects collected from the window trap were alive. 574 (29.9\%) of the live insects 226 and $689(39.9 \%)$ of the dead insects were parous (Chi-Square $10.03 p=0.002)$. Thus, parous 227 insects were more likely to die compared to nulliparous ones. Among the 1281 live nulliparous 228 insects dissected, 567 (44.26\%) were virgins whilst 457 (35.68\%) had mating plugs (Chi-Square $2295.44, p=0.020)$. Among the dead nulliparous insects dissected $311(36.76 \%)$ were virgins and $230366(43.26 \%)$ had mating plugs. The estimated total proportion of the different age groups 231 (combining estimated numbers of both live and dead insects) was also different between 232 mosquitoes collected indoors (light and window-trap combined) and those collected outdoors 233 (Table 3). Virgin insects predominated in the outdoor collection (Chi-Square 16.54. $p<0.001$ ) 234 whilst parous insects, even excluding teneral insects (virgins and those with mating plugs), were 
235 more common indoors (Chi-Square for all insects 41.96, $p<0.001$ and 8.84, $p=0.003$ excluding

236 teneral insects). Hence, newly emerged insects were more likely to attempt to feed outdoors

237 rather than indoors.

238

239 Among parous insects the proportion with ' $a$ ' sacs was significantly greater in insects that were

240 dead $(0.36)$ on collection in the morning compared to those that were alive $(0.12)$ (Chi-Square

241 138.93, $p<0.001$ ) (Figure 5). The estimated duration of the oviposition cycle among live

242 insects, based on equation 1, was 2.7 days and among dead ones was 2.4 days. The proportion of

243 parous insects dissected from Adi Bosco (that were all alive on collection) with large sacs was

244 also significantly different to those from Kyamyorwa (68 of 91 compared to 113 of 424) (Chi-

245 Square 75.97, $p<0.001)$.

246

247 Parous rates were lower in the mosquitoes that had remained alive at the time of capture (Chi-

248 Square $=39.46, p<0.05)$. There was no significant difference in the parous rates of mosquitoes

249 collected in the window trap compared to the light-trap (Chi-Square $=2.57, p=0.109$ n.s.) nor

250 between virgin and plug rates among newly emerged insects from these two types of collection

251 (Chi-Square $=0.0002, p=0.98$. n.s.) but there was a difference between tent and window trap

252 (Chi-Square $=21.76, p=<0.001)$.

253 Discussion

254

255 Reviews of techniques for age determination in insects of medical importance, including the 256 methods used in the present study, have been presented by Charlwood et al., (1980) and Tyndale-

257 Biscoe (1984). Ovaries of Cx. quinquefasciatus were much easier to classify using the dry 258 technique than were the An. arabiensis. The dry technique may therefore be useful for 259 determining the gonotrophic age of this mosquito. Our results indicate, however, that almost 10 
260 per cent of the $A$. arabiensis had unreadable ovaries using this technique, which might affect

261 assessments of survival. Nevertheless, since the discrepancies were almost equally distributed

262 between nulliparous and parous insects an overall estimate of survival would be similar. A

263 similar proportion of unreadable ovaries of Aedes vigilax and Culex annulirostris was observed

264 by Hugo et al. (2008). As with the A. arabiensis this was apparently due to the deposition of

265 material (yolk) in the follicles that obscured the tracheoles. This may particularly be problem

266 with pre-gravid insects that have taken a blood-meal and in which the follicles have advanced to

267 mid to late Stage II. Thus, despite its ease, the dissection of Anopheles ovaries in water and their

268 subsequent examination with a compound microscope when dry, is not as good, or useful, as

269 examination of the ovaries using a stereomicroscope with transmitted light from a mirror. A

270 mirror is better than an artificial light source since by altering its position the contrast of the

271 preparation can be changed so that the visibility of structures within the ovaries changes making

272 assessment easier.

273

274 Results from Tanzania indicate that young A. arabiensis, in particular virgin insects, are more

275 likely to feed outdoors than older ones. This is similar to the behaviour of $A$. coluzzii from Ghana

276 (Charlwood et al., 2012) and indicates that mating has an effect on host seeking in a relatively

277 subtle fashion, at least in the A. gambiae complex but, whether the same behaviour occurs in 278 other endophilic mosquitoes is not known.

279 In many ways young virgin insects act like surrogate males. Thus, they may be more likely to 280 rest in the sites that males do (i.e. outdoors in the case of the A. gambiae complex) compared to 281 older females. Many mosquitoes rest close to where they feed. Host-seeking mosquitoes respond 282 to visual as well as olfactory cues (Bidlingmayer 1975, Bidlingmayer \& Hem, 1979, Hawkes et 
283 al., 2017). Young insects may have different visual priorities (horizontal swarm markers) and

284 may not respond to the silhouette of the house in the same way that older mosquitoes do. This

285 would lead to there being a preponderance of young insects biting outdoors. In the absence of

286 control measures (present throughout most of the insects evolution) there is little mortality

287 among mosquitoes that feed and rest indoors. For virgin insects to spend their time outdoors

288 there must be a survival advantage. It may mean that such insects are more likely to find a mate 289 compared to insects that rest indoors.

290 Whilst a preponderance of young insects biting outdoors might mean that the risk of acquiring 291 malaria per bite is lower outside it also means that the risk of transmitting are greater outdoors 292 since young mosquitoes may be more likely to survive through the extrinsic cycle than older 293 ones. They also imply that a potential control technique aimed specifically at young insects 294 should work preferentially outdoors. Young, naïve mosquitoes may be attracted to a wider range 295 of potential hosts than older insects, which may return to feed on hosts that they have fed on 296 previously (Vantaux et al, 2003, Vinauger et al, 2014). Odour baited traps that target such young 297 insects may be one possible approach.

299 The proportion of live parous mosquitoes with 'a' sacs from the tent-trap recorded from Eritrea 300 was significantly higher than that recorded from Kyamyorwa. The higher rates are probably 301 because the much lower temperatures in Adi Bosco $\left(12^{\circ} \mathrm{C}\right.$ minimum at night in Adi Bosco 302 compared to $27^{\circ} \mathrm{C}$ in Kyamyorwa) slowed contraction of the sacs. At the higher temperatures, 303 typical of the tropics, it behoves the entomologists to kill and dissect the mosquitoes as soon as 304 possible after collection. If there is a delay, sacs are likely to contract during the time that the 305 mosquito is collected and killed. This will tend towards an overestimation of the duration of the 
306 cycle (in our case 2.7 days compared to 2.4 days) and as a result an overestimation of the

307 vectorial capacity of the population as a whole. Given the variation in age and the effect that

308 environmental conditions can have on the relative proportion of the population biting indoors or

309 outdoors (Charlwood et al.,2011) it also behoves the entomologist to undertake simultaneous

310 collections indoors and outdoors for population assessment.

311 Surprisingly, virgins survived better than recently mated insects. This may be because they were

312 collected later in the night than recently mated insects (and so had a shorter time in the stressful

313 environment of the trap). However, given that virgin and recently mated females of $A$. coluzzii

314 have similar patterns of activity in landing collections (Charlwood et al., 2003) and that the rates

315 were similar between light-trap (where the majority of mosquitoes had died) and window-trap

316 (where they were all alive, Le Clair et al, 2017) this is unlikely. Given that it had not been

317 absorbed before dissection, it is unlikely that possible nutritional benefit to the female from the

318 male donated mating plug was available. Under these circumstances the effort involved in

319 mating may have had a detrimental effect on the survival of the female.

320

321 It is possible that dissections will in future be replaced by other techniques, notably assessment

322 of age based on reflectance of Near Infra-Red (NIR) light (Mayagaya et al., 2009, Krajacich et

323 al., 2017) or gene transcription (Cook et al. 2006, 2007). Nevertheless, the techniques remain

324 experimental and in the process of development. For the time being dissections remains the 325 method of choice.

326 Conclusions

327 
328 The utility of examination of tracheolar coiling in dried ovariolar dissections for the assessment

329 of mosquito age differs between genera. Among anophelines the technique is less useful than

330 examination of ovaries wet with transmitted light.

331 The wet dissection also allows for determination of oviposition cycle duration. However, insects

332 need to be dissected shortly after capture for the information to be meaningful.

333 Recently emerged virgin Anopheles arabiensis are more likely to be seek hosts outdoors rather 334 than indoors.

Acknowledgements

337

338

339

We would like to thank the staff of the entomology laboratory of Elaboret for their accommodation and help during the studies in Eritrea and Yohannes Kulwa and the late Mzee Kasege and his family for their help during the work in Tanzania. We also thank Enock Kessey for the identification of the A. gambiae complex mosquitoes from Tanzania. We would like to thank the referees and Editor for their perceptive comments that helped improve the manuscript.

\section{References}

Baldini F, Gabrieli P, South A, Valim C, Mancini F, Catteruccia F. 2013. The interaction between a sexually transferred steroid hormone and a female protein regulates oogenesis in the malaria mosquito Anopheles gambiae. PLoS Biology. 11(10): e1001695.

Bass C, Williamson MS, Field LM. 2008. Development of a multiplex real-time PCR assay for identification of members of the Anopheles gambiae species complex. Acta Tropica 107: 50-53.

Bidlingmayer WL. 1975 Mosquito flight paths in relation to the environment. Effect of vertical and horizontal visual barriers. Annals of the entomological Society of America 68: 51-57.

Bidlingmayer WL, Hem DG. 1979. Mosquito (Diptera: Culicidae) flight behaviour near conspicuous objects. Bulletin of Entomological Research 69: 691-700. 
359 Charlwood JD. 1968 Survival rate variation of Anopheles farauti (Diptera, Culicidae) between 360 neighbouring villages in coastal Papua New Guinea. Journal of Medical Entomology. 23: 361361365.

362

Charlwood JD, Billingsley PF, Takken W, Lyimo EOK, Smith T, Meuwissen JME Th. 1997. Survival and infection probabilities of anthropophagic Anophelines from an area of high prevalence of Plasmodium falciparum in humans. Bulletin of Entomological Research. 87:445453.

Charlwood JD, Birley MH, Dagaro H, Paru R, Holmes PR. 1985 Assessing survival rates of Anopheles farauti (Diptera, Culicidae) from Papua New Guinea. Journal of Animal Ecology. 54: 1003-1016.

Charlwood JD, Birley MH, Graves PM. 1986. Capture-recapture studies of females of the Anopheles punctulatus group of mosquitoes (Diptera, Culicidae) from Papua New Guinea. Bulletin of Entomological Research. 76: 211-227.

Charlwood JD, Paru R, Dagaro H, Lagog M. 1986. The influence of moonlight and gonotrophic age on the biting activity of Anopheles farauti (Diptera, Culicidae) from Papua New Guinea. Journal of Medical Entomology. 23:132-135.

Charlwood JD, Pinto J, Sousa CA, Ferreira C, Gil V, de Rosario V. 2003. Mating does not affect the biting behaviour of Anopheles gambiae from the islands of São Tomé and Príncipe, West Africa. Annals of Tropical Medicine and Parasitology. 97: 751-756.

Charlwood JD, Pinto J, Sousa CA, Ferreira C, Petrarca V, do Rosario VE. 2003. A mate or a meal' - Pre-gravid behaviour of female Anopheles gambiae from the islands of São Tomé and Príncipe, West Africa. Malaria Journal 2: 7

Charlwood JD, Rafael JA, Wilkes TJ. 1980. Metodos de determinar a idade fisiiologica em Diptera de importancia medica. Uma revisão com especial referencia aos vetores de doenças na America do Sul. Acta Amazonica 10: 311-333.

Charlwood JD, Rowland M, Protopopoff N, LeClair C. 2017. The Furvela tent-trap Mk 1.1 for the collection of outdoor biting mosquitoes. PeerJ 3848.

Charlwood JD, Tomás EVE, Salgueiro P, Egyir-Yawson A, Pitts RJ, Pinto J. 2011. Studies on the behaviour of peridomestic and endophagic M form Anopheles gambiae from a rice growing area of Ghana. Bulletin of Entomological Research. 101: 533-539.

Charlwood JD, Tomás EVE. 2011. Do developing malaria parasites manipulate their mosquito host? - Evidence from infected Anopheles funestus (Giles) from Mozambique. Transactions of the Royal Society of Tropical Medicine and Hygiene. 105: 352-354. 
401 Charlwood JD, Tomás EVE, Egyir-Yawson A, Kampango A, Pitts RJ. 2012. Feeding 402 frequency and survival of Anopheles gambiae from a rice growing area of Ghana. Medical \& 403 Veterinary Entomology 26: 263-270.

404

405

406

407

408

409

410

411

412

413

414

415

416

417

418

419

420

421

422

423

424

425

426

427

428

429

430

431

432

433

434

435

436

437

438

439

440

441

442

443

444

445

Clements AN \& Paterson GD. 1981. The analysis of mortality and survival rates in wild populations of mosquitoes. Journal of Applied Ecology 18: 373-399.

Cook PE, Hugo LE, Iturbe-Ormaetxe I, Williams CR, Chenoweth SF, S. A. Ritchie, Ryan PA, Kay BH, Blows MW, O'Neill SL. 2006. The use of transcriptional profiles to predict adult mosquito age under field conditions. Proceedings of the National Academy of Science. U.S.A. 103: $18060-18065$.

Cook PE, Hugo LE, Iturbe-Ormaetxe I, Williams CR, Chenoweth SF, S. A. Ritchie, Ryan PA, Kay BH, Blows MW, O'Neill SL. 2007. Predicting the age of mosquitoes using transcriptional profiles. Nature Protocols 2: 2796-2806.

Detinova TS. 1962. Age-grouping methods in Diptera of medical importance with special reference to some vectors of malaria. Monograph series WHO no 47, 216pp.

Gillies MT. 1956. A new character for the recognition of nulliparous females of Anopheles gambiae Bulletin of the World Health Organization 15: 451-459

Gillies MT. 1989. Anopheline mosquitos: vector behaviour and bionomics. Chapter 16 in Malaria: Principles and Practice of Malariology. Wernsdorfer W.H. \& McGregor I. (eds.) Churchill-Livingstone.

Gillies MT, Coetzee M. 1987. A supplement to the Anophelinae of Africa South of the Sahara (Afrotropical Region) publication no. 55. Johannesburg: South African Institute for Medical Research.

Gillies MT, De Mellion B. 1968. The Anophelinae of Africa South of the Sahara (Ethiopian Zoogeographical Region), 2nd publication no. 54 edn. Johannesburg: South African Institute for Medical Research.

Gillies MT, Wilkes TJ. 1965. A study of the age-composition of populations of Anopheles gambiae Giles and A. funestus Giles in North-Eastern Tanzania. Bulletin of Entomological Research 56: 237-262

Hawkes FM, Dabiré RK, Sawadogo SP, Torr SJ, Gibson G. 2017.Exploiting Anopheles responses to thermal, odour and visual stimuli to improve surveillance and control of malaria. Scientific Reports. 7:17283.

Hoc TQ, Charlwood JD. 1990 Age determination of Aedes cantans using the ovarian oil injection technique. Medical and Veterinary Entomology. 4: 227-233.

Hoc TQ, Wilkes TJ. 1995. The ovariole structure of Anopheles gambiae (Diptera: Culicidae) and its use in determining physiological age. Bulletin of Entomological Research 85: 59-69 
446 Hugo LE, Quick-Miles S, Kay BH, Ryan PA. 2008. Evaluations of mosquito age grading

447

448

449

450

451

452

453

454

455

456

457

458

459

460

461

462

463

464

465

466

467

468

469

470

471

472

473

474

475

476

477

478

479

480

481

482

483

484

485

486

487

488

489

techniques based on morphological changes. Journal of. Medical Entomology 45: 353-369.

Krajacich BJ, Meyers JI, Alout H, Dabiré RK, Dowell FE, Foy BD. 2017. Analysis of near infrared spectra for age-grading of wild populations of Anopheles gambiae. Parasites and Vectors 10: 552.

LeClair C, Cronery J, Kessy E, Tomás EVE, Rowland M, Protopopoff N, Charlwood JD. 2017. 'Repel all borders': Combination mosquito nets enhance collections of endophilic Anopheles gambiae and An. arabiensis in CDC light-traps. Malaria Journal 16: 336

Mayagaya VS, Michel K, Benedict MQ, Killeen GF, Wirtz RA, Ferguson HM, Dowell FE. 2009. Non-destructive determination of age and species of Anopheles gambiae s.1. using Nearinfrared spectroscopy American Journal of Tropical Medicine and Hygiene 81: 622-630.

Romoser WS, Moll RM, Moncayo AC, Lerdthusnee K. 2000. The occurrence and fate of the meconium and meconial peritrophic membranes in pupal and adult mosquitoes (Diptera:

Culicidae). Journal of. Medical Entomology 37: 893-896.

Rosay B. 1961. Anatomical indicators for assessing the age of mosquitoes: the teneral adult (Diptera: Culicidae). Annals of the Entomological Society of America 54: 526-529.

Shililu J, Ghebremeskel T, Mengistu S, Fekadu H, Zerom M, Mbogo C, Guthrie J, Brantly E, Beier JC Novak RJ. 2003. Distribution of anopheline mosquitoes in Eritrea. American Journal of Tropical Medicine and Hygiene 69: 295-302.

Tyndale-Biscoe, M. 1984. Age-grading methods in adult insects: A review. Bulletin of Entomological Research, 74(3), 341-377.

Vantaux A, Lefèvre T, Dabiré KR, Cohuet A. 2014. Individual experience affects host choice in malaria vector mosquitoes. Parasites \& Vectors 7: 24

Vinauger C, Lutz EK, Riffell JA. 2014. Olfactory learning and memory in the disease vector mosquito Aedes aegypti. The Journal of Experimental Biology 217: 2321-2330

Wilkes TJ, Charlwood JD. 1979. A rapid gonotrophic cycle in Chagasia bonneae from Brazil. Mosquito News 39: 137-139.

World Health Organization. 2013. Malaria entomology and vector control. Contents: Guide for tutors - Guide for participants ISBN 978924150581 9. WHO, Geneva. 


\section{Figure 1 (on next page)}

\section{Sac stages of parous mosquitoes}

Sac stages $(a, b, c, d)$ as seen in host-seeking dissected mosquitoes. 'a' and 'b' sacs refer to mosquitoes that are considered to have oviposited a few hours before collection/dissection whilst ' $c$ ' and ' $d$ ' sacs are found in mosquitoes that have delayed returning to feed. $g$ germarium; o- ovariole; s -sac; lo - lateral oviduct. 

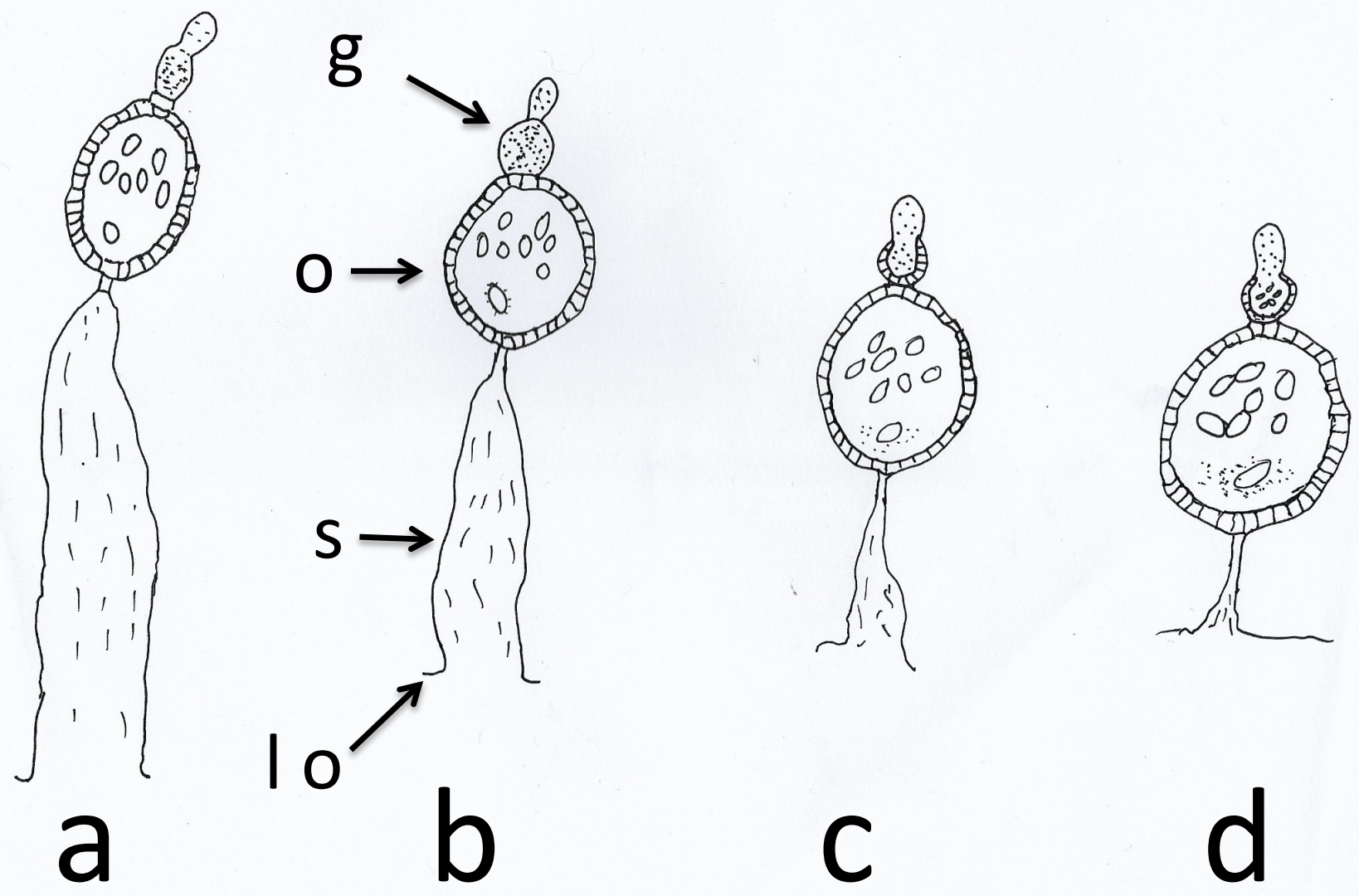
Figure 2

Photograph of dry ovaries of Culex quinquefasciatus

Dry ovaries of Culex quinquefasciatus showing their archetypal, 'textbook' appearance Anulliparous female showing coiled tracheoles; B - parous female showing the tracheoles now streched and uncoiled.
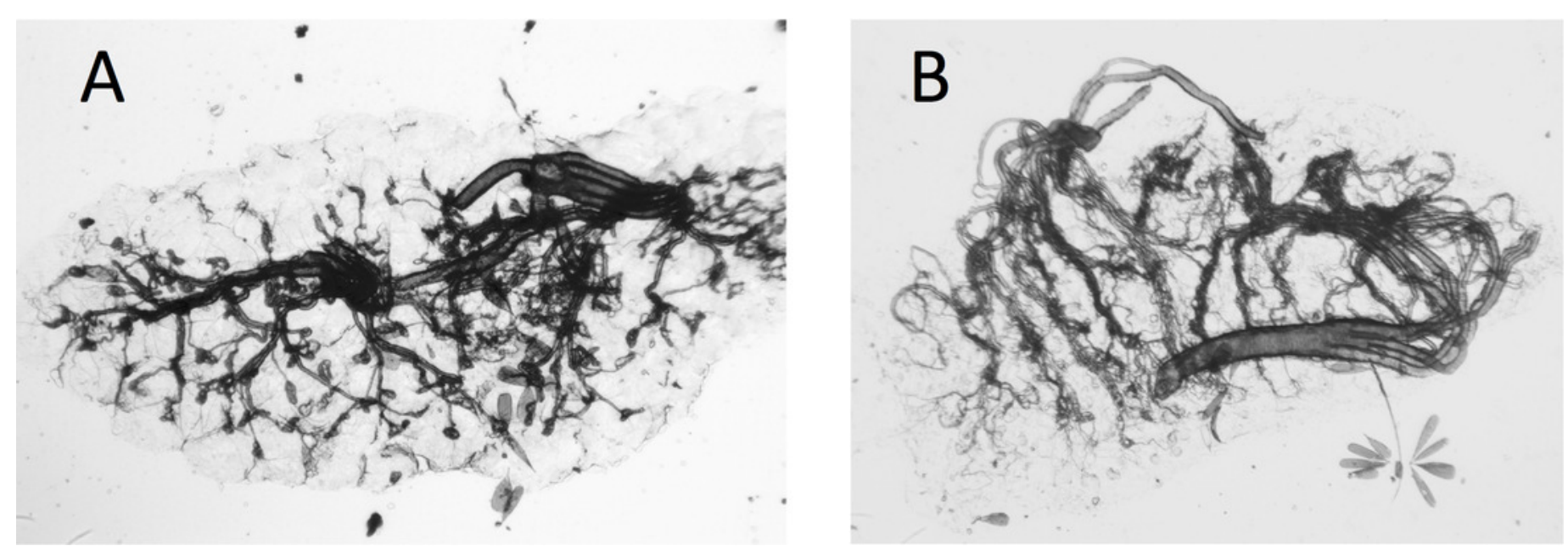
Figure 3

Dry ovaries of Anopheles arabiensis A - nulliparous; B -parous. Compare these with the Cx. quinquefasciatus ovaries in Figure 2 .

Dry ovaries of Anopheles arabiensis A - nulliparous; B -parous. Compare these with the $C x$. quinquefasciatus ovaries in Figure 2.
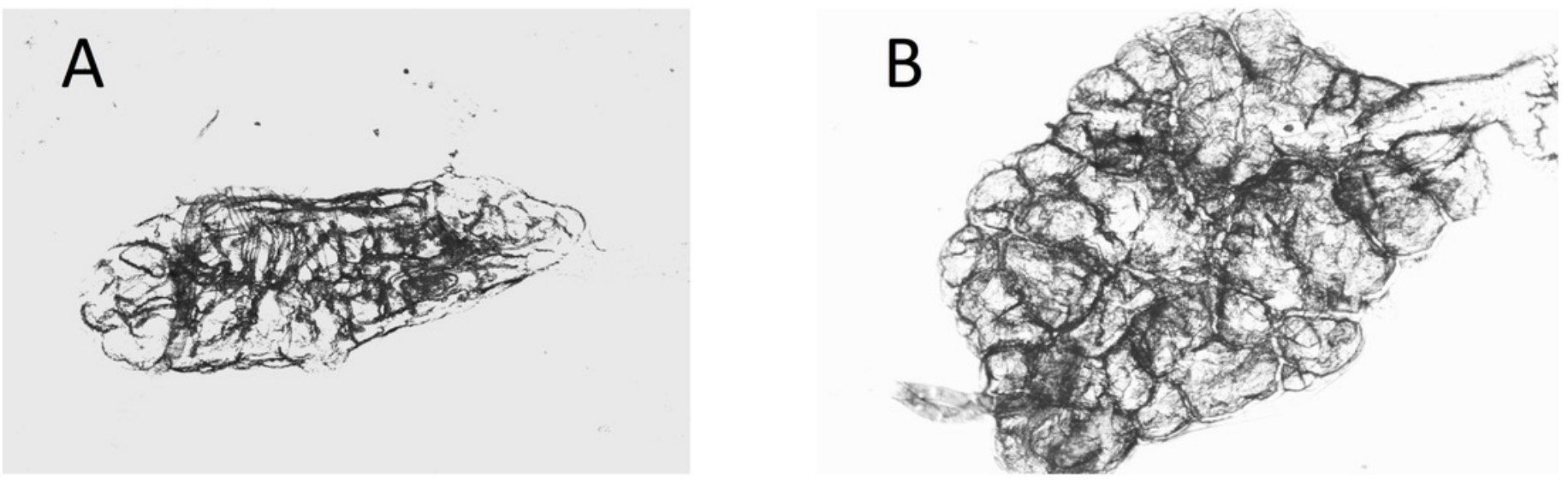


\section{Figure 4}

Ovary of mosquito with yolk A - before and B -after adding water to the dry preparation.

Some dry ovaries that contain yolk (making interpretation difficult) can be temporarily improved by the addition of water to the preparation. The figure shows one such ovary. Adry; B - with water added.
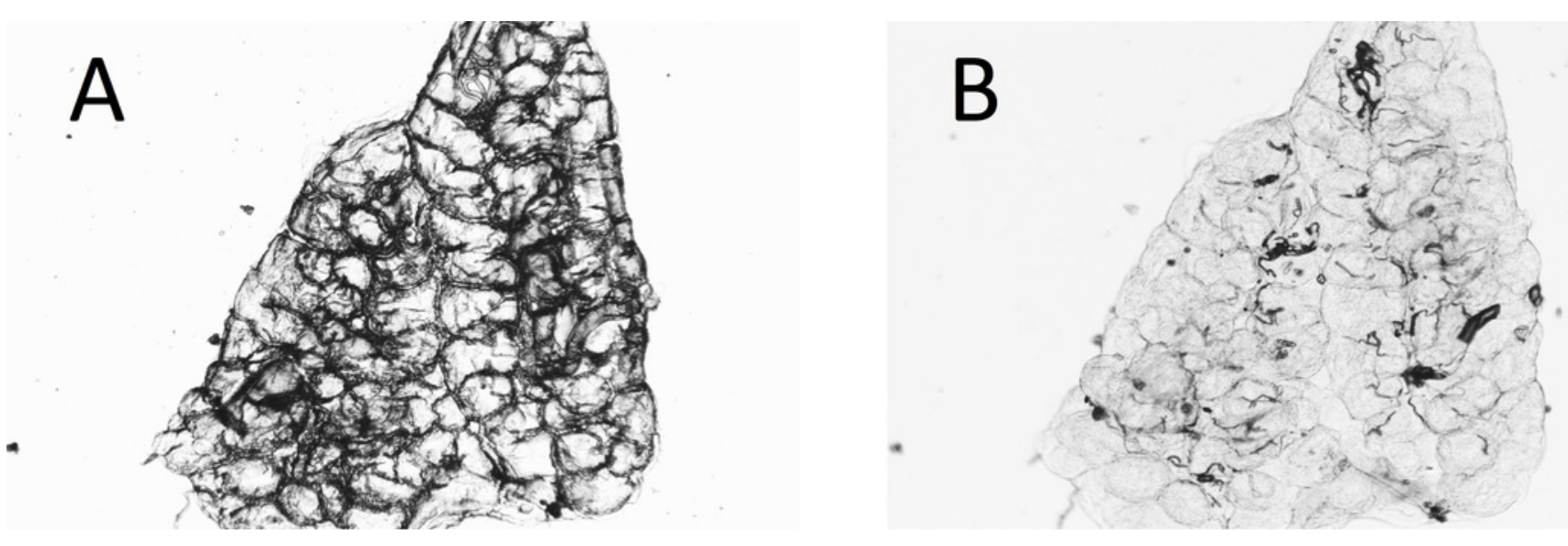


\section{Figure 5 (on next page)}

Sac stages among Anopheles arabiensis that were either alive or dead upon collection

There is an greater proportion of Anopheles arabiensis females with ' $a$ ' or ' $b$ ' sacs among insects that had died upon collection whilst among those that had remained alive the sacs had contracted to ' $c$ ' or ' $d$ ' by the time they were collected. 
0.5

0.4

$\begin{array}{ll}\text { 든 } & \\ \frac{1}{} & 0.3 \\ \frac{1}{} & \\ \text { 응 } & 0.2 \\ \text { 은 } & \\ \text { ․ } & 0.1\end{array}$

0

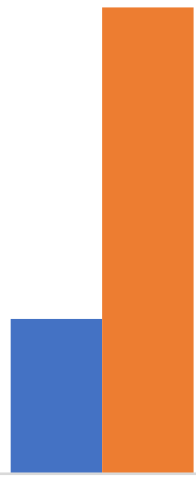

a

b
C

d

Sac stage 


\section{Table $\mathbf{1}$ (on next page)}

Age of Anopheles arabiensis determined either by immediate 'wet' dissection using transmitted light or examined dry with a compound microscope

Age of Anopheles arabiensis from Elaboret, Eritrea, determined either by immediate 'wet' dissection using transmitted light or examined dry with a compound microscope. 
1

2

\begin{tabular}{llccc}
\multicolumn{1}{c}{} & \multicolumn{3}{c}{ Dry dissection } \\
\multirow{4}{*}{ Wet dissection } & Nulliparous & Parous & Total \\
\cline { 2 - 5 } & Nulliparous & 116 & 10 & 126 \\
& Parous & 8 & 77 & 85 \\
\cline { 2 - 5 } & Total & 124 & 87 & 211 \\
\cline { 2 - 5 } & &
\end{tabular}

3 


\section{Table 2 (on next page)}

Number of $A$. arabiensis dissected by age, collection type and mosquito condition (live or dead) on collection.

Note all mosquitoes in the window trap were alive on collection. Virgin - mosquitoes without sperm in the spermatheca; plug - mosquitoes with a mating plug in the common oviduct; $\mathrm{NI}$ nulliparous mosquitoes with sperm in the spermatheca but no mating plug visible and with ovarioles without yolk; NII - nulliparous mosquitoes with sperm in the spermatheca, without a mating plug visible and with ovarioles with yolk present. Adj. Wald C.I. - Adjusted Wald 95\% Confidence Interval. 
1
2
3

3

\begin{tabular}{llcccccccccc}
\hline & & Virgin & Plug & NI & NII & $\begin{array}{c}\text { a- } \\
\text { sac }\end{array}$ & $\begin{array}{c}\text { b- } \\
\text { sac }\end{array}$ & $\begin{array}{c}\text { c- } \\
\text { sac }\end{array}$ & $\begin{array}{c}\text { d- } \\
\text { sac }\end{array}$ & $\begin{array}{c}\text { Total } \\
\text { dissected }\end{array}$ & $\begin{array}{c}\text { Parous rate } \\
\text { (Adj Wald C.I.) }\end{array}$ \\
\hline \multirow{2}{*}{ Tent } & Live & 385 & 321 & 56 & 127 & 32 & 51 & 77 & 160 & 1209 & $0.27(0.25-0.29)$ \\
& Dead & 180 & 167 & 31 & 57 & 94 & 64 & 56 & 56 & 705 & $0.38(0.35-0.42)$ \\
\multirow{3}{*}{ Wight } & Live & 107 & 58 & 6 & 29 & 10 & 7 & 13 & 43 & 273 & $0.27(0.22-0.32)$ \\
& Dead & 131 & 199 & 31 & 50 & 114 & 82 & 64 & 40 & 711 & $0.42(0.39-0.46)$ \\
\hline
\end{tabular}

4

5 


\section{Table 3 (on next page)}

Number of $A$. arabiensis collected indoors (light-trap and window-trap) and outdoors alive or dead on collection and proportion in each age category.

Kyamyorwa, Tanzania, December 2015-January 2016. Prop ${ }^{n}$ - proportion; Adj. Wald C.I. Adjusted Wald 95\% Confidence Interval. * Indoor includes light-trap and window-trap collections combined. Virgin - insects without sperm in the spermatheca; Plug - insects with a mating plug in the common oviduct; Null - All nulliparous insects combined; Parous - all parous insects combined. 


\begin{tabular}{lllllll}
\hline Location & Condition & $\begin{array}{l}\text { Total } \\
\text { collected }\end{array}$ & $\begin{array}{l}\text { Prop }^{n} \text { Virgin } \\
\text { (Adj Wald C.I.) }\end{array}$ & Prop $^{n}$ Plug & Prop $^{n}$ Null & Prop $^{n}$ Parous \\
\hline Indoor* & Live & 560 & $0.27(0.23-0.31)$ & $0.22(0.19-0.26)$ & $0.62(0.58-0.66)$ & $0.38(0.34-0.42)$ \\
& Dead & 3865 & $0.22(0.19-0.25)$ & $0.25(0.23-0.29)$ & $0.58(0.54-0.61)$ & $0.42(0.39-0.46)$ \\
& All & 4425 & $0.22(0.21-0.23)$ & $0.25(0.24-0.26)$ & $0.58(0.57-0.60)$ & $0.42(0.40-0.43)$ \\
Outdoor & Live & 2029 & $0.30(0.28-0.33)$ & $0.27(0.25-0.29)$ & $0.73(0.71-0.75)$ & $0.27(0.25-0.29)$ \\
& Dead & 1605 & $0.24(0.21-0.27)$ & $0.23(0.21-0.26)$ & $0.62(0.59-0.65)$ & $0.38(0.35-0.41)$ \\
& All & 3634 & $0.27(0.26-0.29)$ & $0.25(0.24-0.27)$ & $0.68(0.67-0.70)$ & $0.32(0.30-0.33)$ \\
\hline
\end{tabular}

5 